\title{
Scale Dependency of Total Water Variance and Its Implication for Cloud Parameterizations
}

\author{
Vera Schemann, BJorn SteVens, ANd Verena Grützun \\ Max Planck Institute for Meteorology, Hamburg, Germany \\ JOHANNES QUAAS \\ Universität Leipzig, Institute for Meteorology, Leipzig, Germany
}

(Manuscript received 21 December 2012, in final form 7 June 2013)

\begin{abstract}
The scale dependency of variance of total water mixing ratio is explored by analyzing data from a general circulation model (GCM), a numerical weather prediction model (NWP), and large-eddy simulations (LESs). For clarification, direct numerical simulation (DNS) data are additionally included, but the focus is placed on defining a general scaling behavior for scales ranging from global down to cloud resolving. For this, appropriate power-law exponents are determined by calculating and approximating the power density spectrum. The large-scale models (GCM and NWP) show a consistent scaling with a power-law exponent of approximately -2 . For the high-resolution LESs, the slope of the power density spectrum shows evidence of being somewhat steeper, although the estimates are more uncertain. Also the transition between resolved and parameterized scales in a current GCM is investigated. Neither a spectral gap nor a strong scale break is found, but a weak scale break at high wavenumbers cannot be excluded. The evaluation of the parameterized total water variance of a state-of-the-art statistical scheme shows that the scale dependency is underestimated by this parameterization. This study and the discovered general scaling behavior emphasize the need for a development of scale-dependent parameterizations.
\end{abstract}

\section{Introduction}

The parameterization of cloud processes, including fractional cloud cover, is known to be the principal driver of uncertainties in simulations of climate change (e.g., Bony and Dufresne 2005; Randall et al. 2007). Although different parameterizations use different approaches, all rest on assumptions about the subgrid-scale variability of total water mixing ratio (Tompkins 2008). In this study the scaling behavior of total water variance and its representation in a state-of-the-art parameterization is investigated.

Currently, parameterizations are challenged by a new generation of global climate models (GCMs), which include the possibility to use local grid refinement [e.g., Icosahedral Non-hydrostatic general circulation model (ICON; Wan et al. 2013) and Laboratoire de Météorologie Dynamique general circulation model (LMDZ; Hourdin

Corresponding author address: Vera Schemann, Max Planck Institute for Meteorology, Bundesstr. 53, 20146 Hamburg, Germany. E-mail: vera.schemann@zmaw.de et al. 2006)]. With this it is no longer feasible to tune parameterizations to certain resolutions. The aim has to be to develop parameterizations that adjust naturally to different grid sizes. Those parameterizations should be able to manage a consistent transition from today's GCM resolution $[O(100 \mathrm{~km})]$ down to cloud-resolving scales $[O(1 \mathrm{~km})]$ or even smaller.

According to Arakawa et al. (2011), there are two different approaches to reach this goal. The first approach is to develop parameterizations for a conventional climate model, which are flexible enough to allow for convergence with increasing resolution toward a global cloud-resolving model. An alternative approach is to include a cloud-resolving model as a parameterization in the grid of the global climate model—a so-called "multiscale modeling framework (MMF)."

One promising approach for the first and conventional concept is to develop scale-aware statistical parameterizations. Statistical approaches are based on a representation of the higher moments of the subgrid-scale probability density function (PDF) so as to provide a distribution of the considered quantity — for instance, total water mixing 
ratio. Typically in such approaches a moment expansion is taken and assumptions are required to truncate the moments (e.g., by assuming a given family of PDFs) (e. g., André et al. 1976; Larson and Golaz 2005). Although it is generally well appreciated that the variance should be treated prognostically, the ability to treat higher-order moments is a matter of some debate (e.g., Klein et al. 2005). This motivates the present, as well as other studies, that attempt to understand controls on the subgrid variance, particularly as a function of scale.

Variance as a function of scale can often be described by a power law and with this by a single scaling exponent. By extrapolating the resolved saturation variance with a power-law exponent of $-5 / 3$ and introducing the resulting subgrid-scale variability, Cusack et al. (1999) found an improvement in cloud amount. Since then, a few additional studies have explored how different scales contribute to the water vapor variance. Using aircraft measurements, Cho et al. (2000) found an exponent of $-1.46 \pm 0.04$ for a wavenumber range of approximately $50 \mathrm{~m}-100 \mathrm{~km}$ in the boundary layer; in the free troposphere a higher exponent of $-1.63 \pm 0.05$ for tropical regions and $-1.79 \pm 0.05$ for extratropical regions was estimated. Similar results were found by Kahn and Teixeira (2009), who used data from the Atmospheric Infrared Sounder (AIRS) $(\sim 50-\mathrm{km}$ resolution) to produce a global climatology of scaling exponents for water vapor and temperature. The estimated exponents for water vapor varied between -1.4 and -2.2 . Nastrom et al. (1986) also reported evidence of power-law scaling for water vapor, with a scaling exponent of $-5 / 3$ for the range of approximately $150-500 \mathrm{~km}$ and -2 for $500-1500 \mathrm{~km}$. The height dependency of the exponents is investigated by Fischer et al. (2012), which analyzed a range of 10 $100 \mathrm{~km}$ and found exponents of $-5 / 3$ for the lower troposphere and -2 for the upper level. All these results together imply that there is not a spectral gap between today's resolved $[O(100 \mathrm{~km})]$ and parameterized (less than $\sim 100 \mathrm{~km}$ ) GCM scales and that formulations of the subgrid variance must incorporate information about the scales being parameterized.

Whether or not this scaling continues to the finest scales of variability is more unclear. There is some evidence of a change in scaling, or a scale break, at fine scales. Based on an analysis of numerical weather prediction and climate models, as well as available observational data, Kahn et al. (2011) reported evidence of such a break on scales of order $10 \mathrm{~km}$, with a steeper gradient (less than $-1.8)$ emerging at smaller scales.

All cited studies investigated more details and dependencies on different heights, meteorological conditions, or regions for the scaling of water vapor variance. Here, they are cited in a way to generate a context for the discussion of the results on a more generalized scaling behavior of total water variance. As the differences in the scaling behavior for water-vapor and total water variance are expected to be small, at least for the large scales, a comparison between the previous and the new results can still be useful. The importance of more detailed investigations of the scaling behavior of water vapor for the development of GCM parameterizations was also discussed by Pressel and Collins (2012), who analyzed first-order structure functions in the AIRSobserved water vapor field.

In this study the scaling behavior of total water variance in the warm cloudy boundary layer is analyzed in a hierarchy of models stretching from scales of tens of meters to the global scale. So doing permits the analysis of variance as a function of all relevant scales. The contribution of variance at different length scales is changing across several orders of magnitude. Because the construction of a single composited spectrum spanning all the scales can be sensitive to how the various scales are matched to one another, a detailed analysis is done for each dataset separately and the scaling behavior is analyzed across the individual range of scales spanned by the individual datasets, instead of estimating a universal law for all scales. To further decrease the resulting uncertainty and to provide more background for the discussion of scaling exponents, in this study three different approaches are used for the estimation of the scaling exponent. The question of a scale break is additionally investigated by including data with a resolution of some millimeters from direct numerical simulations (DNS). The scaling behavior identified in this analysis is discussed in the context of the previous studies on water vapor scaling. Furthermore, a state-of-the-art parameterization of subgrid-scale variance developed for use in global climate models is validated against the results obtained in this study.

The manuscript is organized as follows: Section 2 is a short overview of the data and methods used. In section 3 the three different approaches to estimate scaling of total water mixing ratio variance and the respective results are presented. The description of a statistical cloud cover parameterization and its evaluation with regard to the scaling of variance is presented in section 4 . Concluding remarks and perspectives for future research are given in section 5 .

\section{Background}

\section{a. Data}

A continuous analysis of scales ranging from those sufficiently small to resolve ordinary cumulus clouds, to the planetary scales, would require a dataset with very 
high resolution over very large domains. Calculations with this many degrees of freedom (e.g., Satoh et al. 2008) are only just becoming possible, and could be applied to explore these questions using a unified modeling framework, but even these do not reach into the scales relevant for boundary layer clouds. For this reason the present study is based on data from several different model types whose domain size and resolution differ, but which collectively span the desired range of scales.

The University of California Los Angeles (UCLA) Large-Eddy Simulation (LES) model (Stevens et al. 1999, 2005; Stevens and Seifert 2008) is used to investigate the scale dependency of total water mixing ratio variance at cloud-resolving scales. Four different cases with differing grid spacings and domain sizes are investigated. From the Cloud Feedbacks Model Intercomparison Project (CFMIP)/Global Atmospheric System Studies (GASS) Intercomparison of Large-Eddy and SingleColumn Models (CGILS) (Blossey et al. 2013) the tradecumulus case (s6), simulated with a $100-\mathrm{m}$ grid spacing, $96 \times 96$ points, and 128 vertical levels, and the stratocumulus case (s12), simulated with a $25-\mathrm{m}$ grid spacing, $128 \times 128$ points, and 180 vertical levels, were analyzed. For both cases 8 of the 10 simulated days with hourly output were included. In addition, two further 30-h simulations of trade cumulus with hourly output using a grid mesh with $25-\mathrm{m}$ spacing, $1024 \times 1024$ points, and 160 vertical levels were analyzed. The trade-cumulus simulations are based on observations made during the Rain in Cumulus over the Ocean field campaign (RICO) (Rauber et al. 2007), and differ in the specification of free tropospheric humidity following the setup described by Stevens and Seifert (2008). The moister simulation more readily simulates precipitation, which leads to a growth of variance of total water mixing ratio at large scales. More details on the organization and the different distribution of variance can be found in Seifert and Heus (2013).

The scale dependency at the mesoscales $(10-100 \mathrm{~km})$ is assessed using a numerical weather prediction model developed and maintained by the German Weather Service (Deutscher Wetterdienst). Two different setups of this model were explored: the local model Consortium for Small-Scale Modelling Germany (COSMO-DE) with 2.8$\mathrm{km}$ grid spacing and the European version COSMO Europe (COSMO-EU) with 7-km grid spacing (see, e.g., Baldauf et al. 2011). To account for the effects of different physical situations and seasons, several periods of 6-h forecast, each with a length of 4 days, from varying months of 2011 have been analyzed. The two periods (January and June) showing the largest differences were chosen for further study so as to bound the range of behavior evident in the more extensive analysis (not shown).
With simulations using the ECHAM6 GCM (Stevens et al. 2013), larger, through global, scales are analyzed. ECHAM6 is used in two different resolutions: the T63 ( $\sim 200-\mathrm{km}$ resolution at the equator) and the T127 ( 100$\mathrm{km}$ resolution at the equator) spectral truncations. Because the area of each grid box of the latitude-longitude grid corresponding to the GCM spectral resolution converges toward the poles, the analysis is limited to the tropics, defined as the region between $30^{\circ} \mathrm{N}$ and $30^{\circ} \mathrm{S}$ so as to have grid boxes of approximately equal size. Within the analyzed region the distance between two longitudes varies between 111 and $96 \mathrm{~km}$ for the T127 grid, or twice that for the coarser-resolution version of ECHAM6. Hence, the transform (physical space) grid is relatively uniform. In the analysis that follows the wavenumber spectrum is not corrected for the remaining small inhomogeneities in the grid relative to a fixed mesh with a spacing of $100 \mathrm{~km}$ (respectively $200 \mathrm{~km}$ ). One month of data (June) with instantaneous output every $6 \mathrm{~h}$ is analyzed.

In addition to the three types of models used for the main analysis, data from DNS are included to investigate the question of a scale break at finest scales. The DNS data were obtained from a $(1024)^{3}$ simulation of a 3-m-wide mixed-layer configuration that mimics a stratocumulus top solely driven by evaporative cooling (Mellado 2010). The power density spectra from four different heights are included. As those domain sizes and resolutions are still negligible for global parameterizations, these data are not included in the main analysis but provide information on the issue of a possible scale break.

To further simplify matters, analysis is restricted to grid levels where warm clouds can be found. This facilitates a comparison of models that span a wide range of resolutions, as finescale simulations incorporating processes related to ice clouds are not available to us. Because of the diversity of models used, it was necessary to define a general condition as to decide which model levels are related to warm clouds, and thus should be incorporated into the analysis. A good indicator for warm clouds is the presence of liquid water. Hence the vertical profiles (average over time and space) of liquid cloud water mixing ratio was used to identify levels relevant to warm clouds, and those levels where the liquid water was within $10 \%$ of the value at the level containing the maximum liquid water were analyzed (e.g., see Fig. 1).

\section{b. Method}

\section{1) FOURIER TRANSFORMATION}

The Fourier transformation requires periodic input data. If this requirement is not fulfilled the spectrum may show some artifacts associated with mismatches at the end 


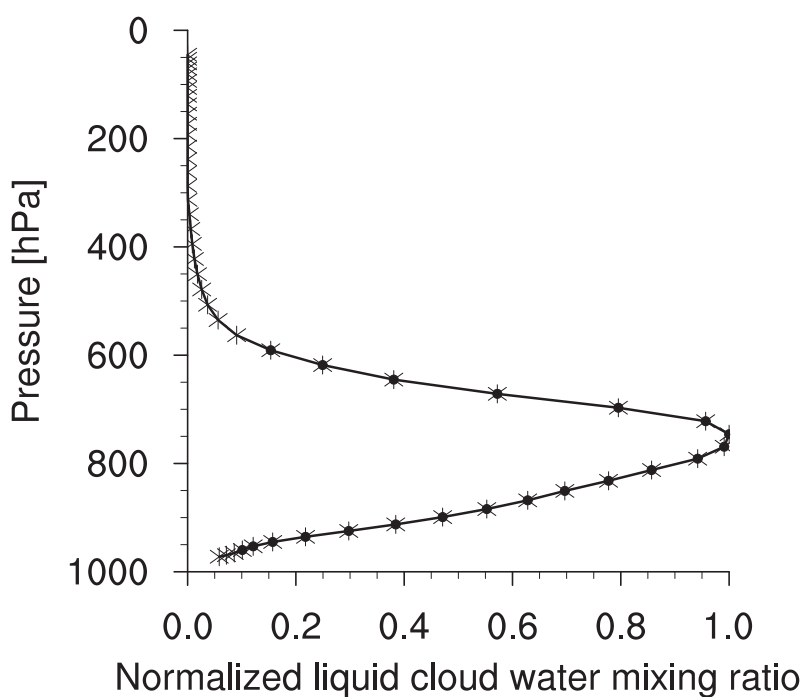

FIG. 1. Temporal- and domain-average vertical profile of cloud liquid water mixing ratio for the COSMO-DE June dataset, normalized by its maximum value. Dots represent the mean values for the model levels and asterisks the levels selected (i.e., those with a value larger than the threshold of 0.1 ).

points. As the COSMO-DE, the COSMO-EU, and the ECHAM6 datasets are not doubly periodic, it is necessary to preprocess the data by removing any linear trends and tapering the data records to remove end-point effects. For the tapering, a split-cosine-bell tapering is applied, following the implementation in the National Center for Atmospheric Research (NCAR) Command Language (NCL 2012). For consistency, tapering was also applied to the datasets with periodic boundary conditions (LES, GCM in longitude direction).

The two-dimensional discrete Fourier transform is defined as

$$
\begin{aligned}
\mathcal{F}(k, l) & =\frac{1}{M N} \sum_{m=0}^{M-1} \sum_{n=0}^{N-1} f(m, n) e^{-i 2 \pi(m k / M)} e^{-i 2 \pi(n l / N)} \\
& =\frac{1}{M N} \sum_{m=0}^{M-1} \sum_{n=0}^{N-1} f(m, n) e^{-i 2 \pi[(m k / M)+(n l / N)]},
\end{aligned}
$$

with the wavenumbers $k=0, \ldots, M-1, l=0, \ldots$, $N-1$, the wavenumber-dependent Fourier coefficients $f(m, n)$, and

$$
\begin{aligned}
e^{-i 2 \pi[(m k / M)+(n l / N)]}= & \cos \left[2 \pi\left(\frac{m k}{M}+\frac{n l}{N}\right)\right] \\
& -i \sin \left[2 \pi\left(\frac{m k}{M}+\frac{n l}{N}\right)\right] .
\end{aligned}
$$

The two-dimensional discrete Fourier transform can also be rewritten and calculated as a twice-implemented one-dimensional Fourier transform:

$$
\mathcal{F}(k, l)=\frac{1}{N} \sum_{n=0}^{N-1}\left[\frac{1}{M} \sum_{m=0}^{M-1} f(m, n) e^{-i 2 \pi(m k / M)}\right] e^{-i 2 \pi(n l / N)} .
$$

The resulting two-dimensional discrete Fourier transform is symmetric around the origin, which means $|\mathcal{F}(k, l)|=|\mathcal{F}(-k,-l)|$. Because of this, it is common to describe the two-dimensional Fourier transform centered around the mean value, with wavenumbers $-\lfloor M / 2\rfloor \leq$ $k \leq\lfloor(M-1) / 2\rfloor,-\lfloor N / 2\rfloor \leq l \leq\lfloor(N-1) / 2\rfloor$.

The effective frequency of the two-dimensional discrete Fourier transform is defined as

$$
\hat{f}_{(k, l)}=\frac{1}{\Delta} \sqrt{\left(\frac{k}{M}\right)^{2}+\left(\frac{l}{N}\right)^{2}}
$$

where $\Delta$ is the spatial resolution. For this definition it is assumed that the spatial resolution is the same in both directions, which means $\Delta x=\Delta y=\Delta$. This is true for the LES and numerical weather prediction (NWP) datasets and, as discussed previously, can also be assumed for the analyzed ECHAM6 region. To avoid aliasing and artifacts, the effective frequency has to be constrained on $\hat{f}_{k, l} \leq 1 /(2 \Delta)$ for all $k$ and $l$.

\section{2) Power Density SPECTRUM}

The power density spectrum of the two-dimensional Fourier transform provides scale-by-scale information about the variance and the variance scaling. It is defined as the product of the Fourier transform and its complex conjugate. For instance, if $\mathcal{F}$ is the discrete Fourier transform, the power spectrum $\mathcal{P}$ is defined by

$$
\mathcal{P}=\mathcal{F} \mathcal{F}^{*}=\left[\Re(\mathcal{F})^{2}+\Im(\mathcal{F})^{2}\right],
$$

where $\mathcal{F}^{*}$ is the complex conjugate, $\Re(\mathcal{F})$ is the real and $\Im(\mathcal{F})$ is the imaginary part of $\mathcal{F}$.

The power density at each point includes the information about the variance at a certain effective frequency or wavenumber. If the spectrum is isotropic then it is possible to reduce the two-dimensional $(k, l)$ power spectrum into a one-dimensional spectrum by integrating over annuli that define an effective frequency. In the present study this is accomplished by binning the data by effective frequency. The number of bins can also be used to smooth the resulting spectrum. In the following results $\left\lfloor(3 / 4) \times n_{p}\right\rfloor$ bins were chosen, where $n_{p}$ is the minimum of the number of discrete wavenumbers in both spectral directions. With this the range of bins is defined as $[0, \Delta k, \ldots, 1 /(2 \Delta)]$, where $\Delta$ is the grid spacing of the original data and 


$$
\Delta k:=\frac{1}{3 / 4 \times n_{p} \times \Delta} .
$$

To get a quantity that is independent of the bin width $(\Delta k)$, the density is calculated by dividing by $\Delta k$.

In the following analysis the focus will be placed on the scaling behavior and not the amount of variance. For this the normalized power density spectrum is of interest and is defined by

$$
p\left(k_{i}\right)=\frac{\mathcal{P}\left(k_{i}\right)}{\Delta k} \frac{1}{\sum_{k_{j} \in K} \mathcal{P}\left(k_{j}\right)},
$$

where $K$ includes all considered wavenumbers and $k_{i} \in K$.

By Parseval's theorem the integral over the whole power spectrum is equal to the total resolved variance. In the discrete sense this means

$$
\sigma^{2}=\sum_{k_{j} \in K} \mathcal{P}\left(k_{j}\right)
$$

where $\sigma$ is the standard deviation.

To calculate the variance, which is resolved with a certain range of wavenumbers $k_{1} \leq k \leq k_{2}$, it is necessary to integrate the discrete spectrum between the minimum wavenumber $k_{1}$ and the maximum wavenumber $k_{2}$ :

$$
\sigma^{2}\left(k_{1}, k_{2}\right)=\sum_{k=k_{1}}^{k_{2}} \mathcal{P}(k) .
$$

\section{3) LEAST SQUARES FIT}

To estimate the slope of the power spectrum a least squares algorithm is applied. If the data follow a power law, they fulfill the following equation:

$$
\begin{aligned}
\mathcal{P}\left(k_{i}\right) & \approx \beta k_{i}^{\alpha}, \quad i=1,2 \ldots \\
\ln \left[\mathcal{P}\left(k_{i}\right)\right] & \approx \ln (\beta)+\alpha \ln k_{i}, \quad i=1,2 \ldots
\end{aligned}
$$

The coefficients $\alpha$ and $\beta$, which fit the data with respect to the least squares constraint, are defined implicitly by minimizing the function

$$
F(\alpha, b)=\sum_{i=1}^{n}\left(y_{i}-b-\alpha x_{i}\right)^{2}
$$

\begin{tabular}{|c|c|c|c|c|}
\hline Data & $k_{\max }^{-1}(\mathrm{~m})$ & $k_{\min }^{-1}(\mathrm{~m})$ & $\begin{array}{l}\text { Time } \\
\text { steps }\end{array}$ & Levels \\
\hline LES (CGILS s12) & 59.38 & 237.50 & 191 & 56 \\
\hline LES (CGILS s6) & 236.67 & 946.67 & 191 & 61 \\
\hline $\begin{array}{l}\text { LES (RICO moist } \\
\text { low } k \text { ) }\end{array}$ & 255.67 & 2556.67 & 26 & 74 \\
\hline $\begin{array}{l}\text { LES (RICO moist } \\
\text { high } k \text { ) }\end{array}$ & 63.92 & 153.40 & 26 & 74 \\
\hline $\begin{array}{l}\text { NWP (COSMO-DE } \\
\text { January) }\end{array}$ & 13526.15 & 175840.00 & 33 & 20 \\
\hline $\begin{array}{l}\text { NWP (COSMO-DE } \\
\text { June) }\end{array}$ & 13526.15 & 175840.00 & 33 & 21 \\
\hline $\begin{array}{l}\text { NWP (COSMO-EU } \\
\text { January) }\end{array}$ & 27496.00 & 343699.97 & 33 & 15 \\
\hline $\begin{array}{l}\text { NWP (COSMO-EU } \\
\text { June) }\end{array}$ & 27496.00 & 343699.97 & 33 & 14 \\
\hline GCM (ECHAM6 T63) & 460000.00 & 2300000.00 & 120 & 13 \\
\hline GCM (ECHAM6 T127) & 235000.02 & 2350000.00 & 120 & 13 \\
\hline
\end{tabular}

where $n$ is the number of considered points, $y_{i}=$ $\ln \left[\mathcal{P}\left(k_{i}\right)\right], b=\ln (\beta)$, and $x_{i}=\ln \left(k_{i}\right)$.
TABLE 1. The investigated intermediate range, number of time steps, and number of levels for each dataset.

\section{Scaling of variance \\ a. Estimation of a valid range}

The power density spectra for the whole wavenumber space is calculated, but only an intermediate range is considered robust because of numerical and subgrid parameterization effects at small scales (high wavenumbers) and domain size constraints, which influence the largest scales, or lowest wavenumbers. For this reason focus is placed on the calculation of power-law exponents for an intermediate range of wavenumbers for each model. But also in this intermediate range there might be some fluctuations or a transition between different power-law exponents. To address this issue an attempt is made to identify if there is an intermediate range of wavenumbers over which a power-law scaling is evident. Ideally, within this range the estimated exponent is not sensitive to the exact start and end point of the chosen range. When power-law scaling is not evident the estimated exponent will depend on the start and end points of the analyzed range and it will not be reasonable to estimate a single exponent.

A procedure as described below helps to find a good estimate for a robust intermediate range by revealing dependencies of the exponent on the exact start and end points more clearly. Nevertheless, the method is not purely objective and for each dataset a decision about acceptable deviations and uncertainties has to be made. The intermediate range for each dataset chosen for this study, as well as the number of levels and time steps investigated, can be found in Table 1.

For a particular model the power density spectrum over the whole wavenumber space at each time and at 


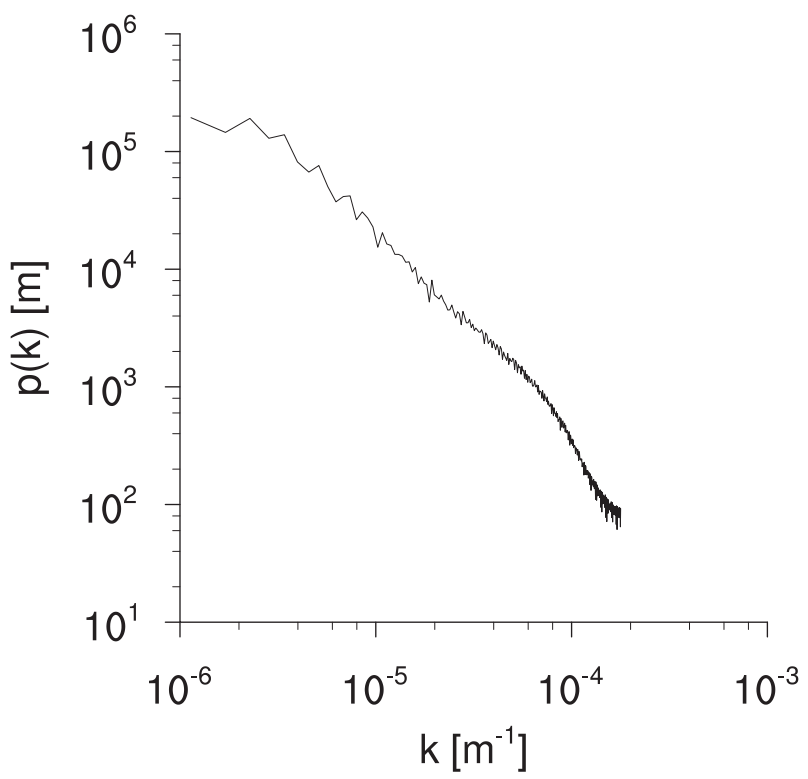

FIG. 2. Normalized power density spectrum of total water mixing ratio for the COSMO-DE June dataset, averaged over time and height.

each level is first normalized to unit variance and then averaged across all the levels and time periods analyzed to produce a mean spectrum. An example of such a spectrum is shown in Fig. 2 for the case of the COSMO-DE model. By eye the spectrum shows evidence of three regimes. The power density spectrum is relatively flat at very low wavenumbers, has apparent power-law scaling in an intermediate range, and then the spectrum falls off increasingly sharply at high wavenumbers. The following procedure aims at identifying a minimum and maximum wavenumber, which sets limits to the intermediate range that shows a robust power-law scaling.

The identification of the minimum and maximum wavenumber is outlined using the example of the COSMO-DE June dataset, which is shown in Fig. 3. To determine the minimum wavenumber, a wavenumber in the middle of the possible range for each model domain [e.g., $k_{\text {end }} \approx 1 /(7 \Delta)$ ] is chosen as a fixed end point and the start wavenumber $\left(k_{\text {start }}\right)$ is increased from $k_{1}$ until $k_{\text {end. }}$. For each $k_{\text {start }}$ a least squares fit between $k_{\text {start }}$ and $k_{\text {end }}$ is performed, which gives an estimate for the exponent. The dependence of the estimated exponents on the choice of $k_{\text {start }}$ is shown in Fig. 3a. At high wavenumbers clear fluctuations can be seen, as the number of points used for the least squares fit is decreasing with increasing $k_{\text {start }}$. Where the line in Fig. 3a is approximating a straight line, the calculated exponent becomes less dependent on the exact starting point $\left(k_{\text {start }}\right)$ and with this a good estimate for the start of a robust power law is reached. To find the maximum wavenumber the procedure
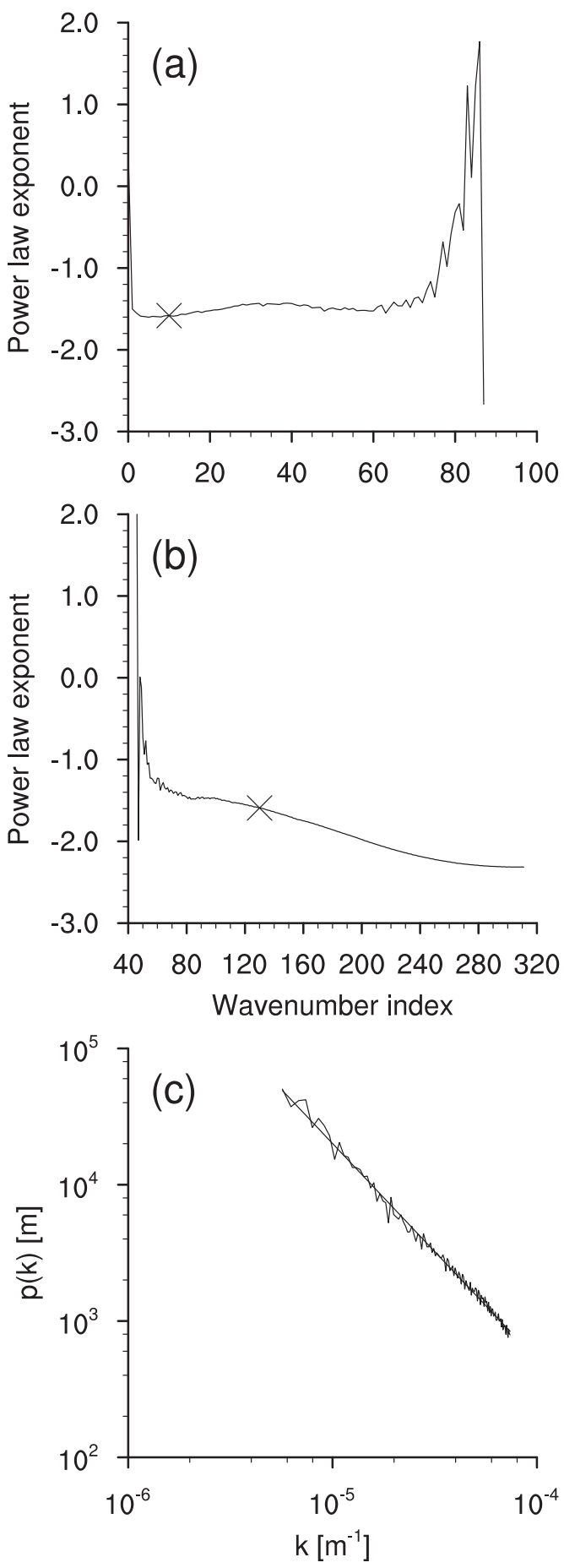

FIG. 3. Estimation of the robust range for a power law for the COSMO-DE June dataset by approximating the power density spectrum of total water mixing ratio. The variability of the powerlaw exponent due to a change in the (a) minimum and (b) maximum wavenumber of the fitted wavenumber range. Small changes represent the beginning (or end) of a stable power-law range. The chosen wavenumber index is marked with a cross. (c) A least squares fit (with a slope of $\alpha \approx-1.6$ ) to the selected range. 


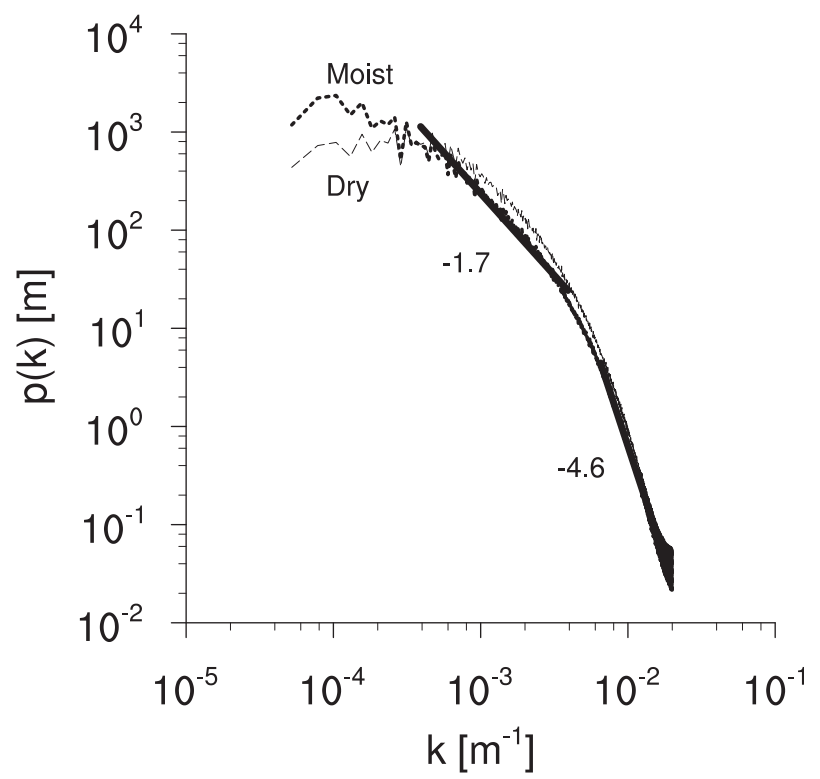

FIG. 4. Normalized power density spectrum of total water mixing ratio for the two RICO datasets, averaged over time and height for the moist (thick dotted) and dry (thin dashed) cases of the tradecumulus case simulated at $25-\mathrm{m}$ resolution. Two fits to the moist RICO case are shown: one for the high wavenumbers $(-4.6)$ and one for the low wavenumbers $(-1.7)$.

is repeated the other way around: a start wavenumber is fixed and from this on $k_{\text {end }}$ is increased until the highest possible value is reached (Fig. 3b). Here the fluctuations due to a too-small number of points can be seen at low wavenumbers, as the number of points used for the least squares fit is increasing by increasing $k_{\text {end }}$.

In this procedure there are two choices, which have to be made empirically: first, the fixed start and end point and, second, the threshold for a robust behavior. The fixed start and end point should already be in the stable range but for a reasonable estimate with a least squares fit as many points as possible are needed. Because of these constraints the choice for the exact start and end points are made depending on the model and the respective stable range. The exponents calculated by changing the start or end point of the least squares fit should approximate a straight line for a robust powerlaw behavior. But there will always remain some fluctuations and a threshold for robust behavior has to be defined for each model independently. The made choices can be tested by looking at the resulting fit for an intermediate range. For the COSMO-DE June dataset this fit is shown in Fig. 3c.

For the two RICO cases, the procedure has been slightly extended. Both RICO cases show a similar behavior for the high, but differ for the low wavenumbers. Figure 4 shows the normalized and averaged power density spectrum for both RICO cases. For the high

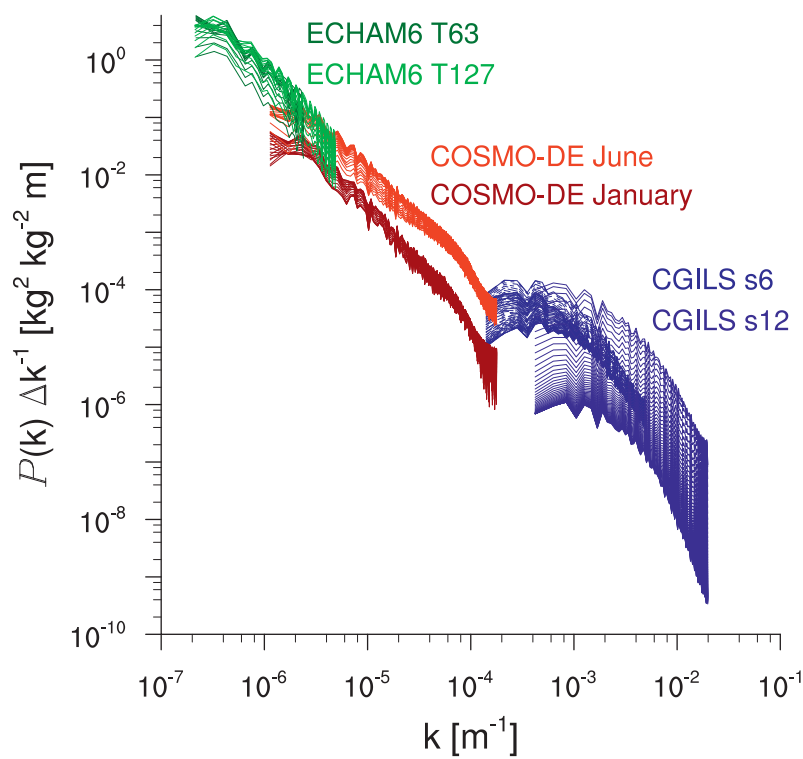

FIG. 5. Power density spectra of total water mixing ratio for the GCM- (ECHAM6), NWP- (COSMO-DE), and CGILS LES. Different lines of the same color represent different height levels. The spectra are temporally averaged.

wavenumbers both show a steep gradient with a scaling exponent around -4.6 . But for the large scales (low wavenumbers) the moist RICO case shows a growth in variance while the dry RICO case is already flat. As both cases have a similar scaling behavior at high wavenumbers, but only the moist RICO case shows a scaling at low wavenumbers, only this case will be used for a more detailed estimation of the scaling exponent. To evaluate both scaling gradients the dataset is split up into two parts: one part consisting of the range of high wavenumbers and one part consisting of the range of low wavenumbers.

\section{b. Power density spectra of total water mixing ratio}

Figure 5 shows datasets from all three kinds of models (GCM, NWP, and LES), simulated for different regions and different seasons. This figure, showing the whole wavenumber range, demonstrates how well the datasets connect to each other and gives the impression of a continuous scaling. As the lines for each model are quite parallel and only shifted on the $y$ axis, there is no evidence for a height dependency of the scaling exponents but for the total amount of variance. Even though some variability in the slopes of differing models can be seen, there is a continuous distribution of variance from the large scales of GCMs $[O(100-1000 \mathrm{~km})]$ until small scales of an LES $[O(100 \mathrm{~m}-10 \mathrm{~km})]$ without evidence for a spectral gap.

Having ruled out the possibility of a spectral gap (i.e., a range of scales with greatly diminished variance), focus 


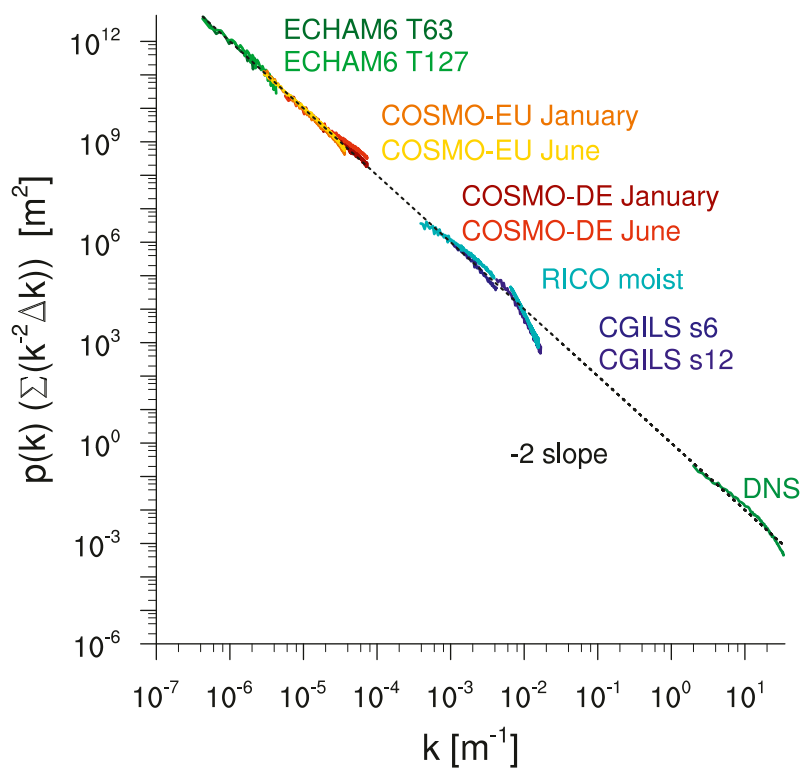

FIG. 6. Power density spectra of the intermediate wavenumber space of total water mixing ratio. The integral over the spectra of each model is adjusted to the integral of a $k^{-2}$ slope. The adjusted spectra are averaged over time and height.

is placed on the scaling (i.e., the slopes of the individual spectra in Fig. 5). For a close look on the consistent scaling the mean variance (integral over the intermediate wavenumber space) of each model is adjusted to the variance of a $k^{-2}$ slope (see Fig. 6). This calculation only affects the height of each line, not the slope. Especially the large-scale models (GCM and NWP) show a similar slope and a scaling close to -2 . This finding holds down to the range of the s6 CGILS case and the larger scales of the RICO dataset. For the small LES scales the gradient becomes steeper. This change may hint at a scale break around $1 \mathrm{~km}$. This result would be in a similar range like the finding of Kahn et al. (2011) for water vapor. To investigate the question of a possible scale break in more detail, in this part of the evaluation also some data from DNS are included. As these data also show a scaling around -2 , a strong break in the scaling, with a change in the exponent of $50 \%$ or more, is not evident. The steeper gradient at the high wavenumbers (approximately $k>4 \times 10^{-3} \mathrm{~m}^{-1}$ ) for the LES might also be due to too much dissipation at the small scales in the model. But because of the variability a weak scale break is certainly possible.

To explore these issues in more depth, Fig. 7 shows the compensated spectrum. The compensated spectrum is calculated by adjusting the mean variance to the $k^{-2}$ slope and an additional scaling by $k^{-2}$. Over a large range of scales, a consistent scaling can be seen, but also some deviations are obvious. There are some fluctuations for the large-scale models around a scaling of -2

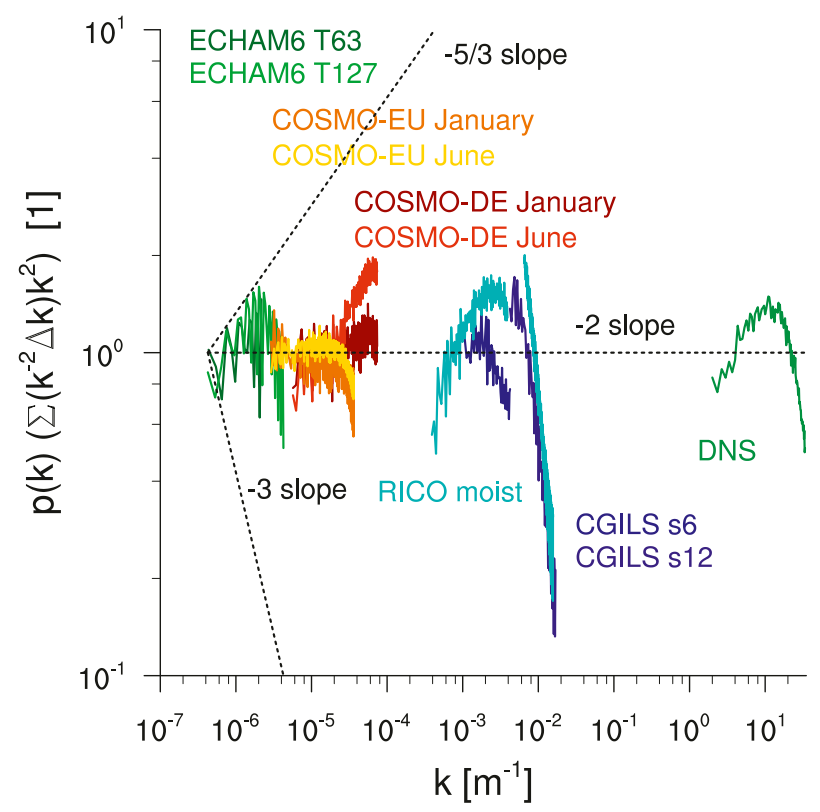

FIG. 7. As in Fig. 6, except the whole spectrum is additionally scaled by $k^{-2}$ (compensated power density spectrum).

and this variability increases with the wavenumber. Especially the LES and DNS data tend to show a $-5 / 3$ scaling at the lower bound of their wavenumber range and an increasing exponent at the higher bound. By this analysis a weak scale break toward a $-5 / 3$ or also a $-7 / 3$ scaling at the meso- and smaller scales [i.e., $O(1-10 \mathrm{~km})$ or smaller] cannot be ruled out.

This first evaluation of the power density spectra shows a broadly consistent scaling among the different models and gives a first estimate for the power-law exponent $(\alpha \approx-2)$. Additionally, there is no clear evidence of a strong break of scales in the spectrum, although a weak change with a transition to a slightly flatter $(-5 / 3)$ or steeper $(-7 / 3)$ spectra is difficult to rule out. In the following sections a more detailed estimation for the power-law exponent is presented.

\section{c. Estimation of the power-law exponents based on least squares fit}

To describe the scaling of the variance of total water mixing ratio in more detail, a good estimate for the power-law exponent is necessary. One possibility to get an estimate is to use the least squares fitting algorithm, described in section $2 b(3)$. With this not only a general estimate for the mean power density spectrum (as in section $3 \mathrm{~b}$ ) is possible, but also an estimate for every level and every time step can be calculated. The resulting histograms of power-law exponents (see Fig. 8) also describe the spread in the estimates and show the variability within the different datasets. A narrow histogram (e.g., COSMO-DE and -EU, or ECHAM6) provides 

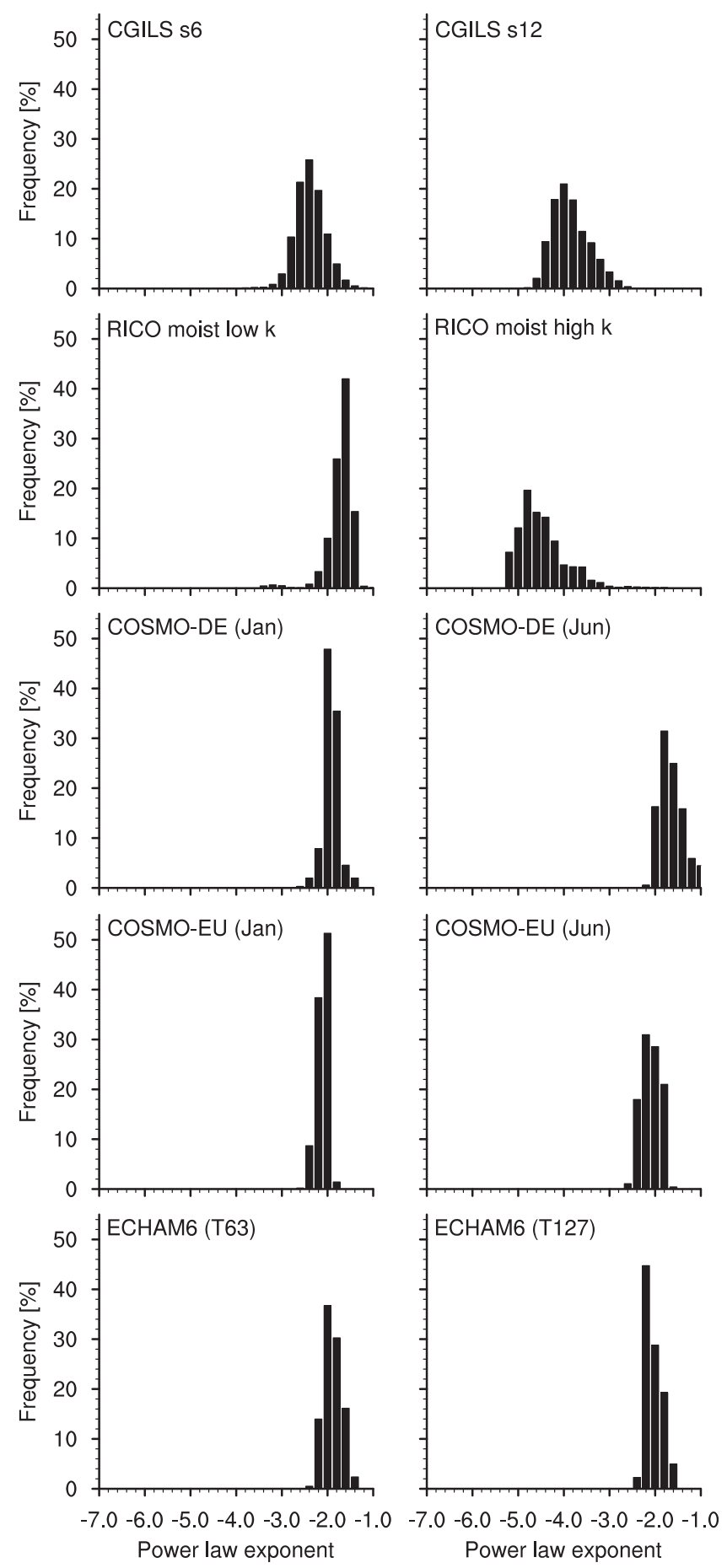

FIG. 8. Histograms of power-law exponents estimated by least squares fitting done individually for each height level and time step of the model simulations.

evidence of a robust power law and only small variability, while a broad histogram (e.g., CGILS s12) hints at deviations from a robust power-law scaling or at a high variability.
The impression of a robust estimate for the large-scale models is supported by calculation of the mean exponents (see Table 2). The NWP and GCM datasets have similar mean values with a small standard deviation of 
TABLE 2. Power-law exponents estimated by a least squares fit, as mean values and standard deviation for all time steps and levels considered.

\begin{tabular}{lcc}
\hline \hline \multicolumn{1}{c}{ Data } & $\begin{array}{c}\text { Mean } \\
\text { exponent }(\alpha)\end{array}$ & $\begin{array}{c}\text { Standard } \\
\text { deviation }\end{array}$ \\
\hline LES (CGILS s12) & -3.85 & \pm 0.40 \\
LES (CGILS s6) & -2.38 & \pm 0.33 \\
LES (RICO moist low $k$ ) & -1.72 & \pm 0.28 \\
LES (RICO moist high $k$ ) & -4.52 & \pm 0.55 \\
NWP (COSMO-DE January) & -1.92 & \pm 0.16 \\
NWP (COSMO-DE June) & -1.65 & \pm 0.26 \\
NWP (COSMO-EU January) & -2.11 & \pm 0.12 \\
NWP (COSMO-EU June) & -2.10 & \pm 0.21 \\
GCM (ECHAM6 T63) & -1.89 & \pm 0.19 \\
GCM (ECHAM6 T127) & -2.04 & \pm 0.18 \\
\hline
\end{tabular}

about $10 \%$ of the average. The overall mean value for the large-scale models $\alpha=-1.94 \pm 0.24$ is close to the first estimate of -2 from the previous section. Also in this evaluation the LES datasets show a differing behavior for the high wavenumbers. The gradients are steeper and also the spread is increasing in absolute terms. The increasing variability in the estimate provides further evidence for the previous conclusion: that the steeper gradient for the high-resolved LES is likely not a signature of a scale break but rather is associated with numerical issues (e.g., too much dissipation).

\section{d. Estimation of the power-law exponents based on extrapolation}

The approach for estimating the power-law exponents used in this section is based on a method described by Cusack et al. (1999), where it was used to estimate the amount of unresolved variance by assuming a certain power law and extrapolating. Some modifications to the original method are necessary in order to use it for the estimation of a power-law exponent.

First, the wavenumber space is divided into two subsets of wavenumbers higher and lower than a chosen threshold: the so-called unresolved $\left\{k_{\text {unres }}\right\}$ and resolved $\left\{k_{\text {res }}\right\}$ part. Analogously the total variance can be divided into two parts with contributions from the resolved (low wavenumbers) and unresolved (high wavenumbers) wavenumber range:

$$
\begin{aligned}
& \sigma_{\text {tot }}^{2}=\sigma_{\text {res }}^{2}+\sigma_{\text {unres }}^{2} \\
& \Leftrightarrow \sum_{k_{x} \in k_{\mathrm{tot}}} \sum_{k_{y} \in k_{\mathrm{tot}}} \mathcal{P}\left(k_{x}, k_{y}\right)=\sum_{k_{x} \in k_{\mathrm{res}}} \sum_{k_{y} \in k_{\mathrm{res}}} \mathcal{P}\left(k_{x}, k_{y}\right) \\
& +\sum_{k_{x} \in k_{\text {urres }}} \sum_{k_{y} \in k_{\text {unres }}} \mathcal{P}\left(k_{x}, k_{y}\right) \text {. }
\end{aligned}
$$

Now the assumption of having a power law is used. In the first approach (section 3c), where the powerlaw exponent $\alpha$ has been introduced, the data were integrated over annuli defined by effective frequency. This time every combination of $\left(k_{x}, k_{y}\right)$ is allowed to contribute to the calculated variance. As the number of modes $\left(k_{x}, k_{y}\right)$ in one annulus scales with the radius $\left(k=\sqrt{k_{x}^{2}+k_{y}^{2}}\right)$, an exponent of $\alpha-1$ is used in the following equations. For details see Cusack et al. (1999).

The ratio of total and resolved variance can then be written as

$$
\begin{aligned}
& \frac{\sigma_{\mathrm{tot}}^{2}}{\sigma_{\mathrm{res}}^{2}} \approx \frac{\sum_{k_{x} \in k_{\mathrm{tot}}} \sum_{k_{y} \in k_{\mathrm{tot}}} \psi\left(\sqrt{k_{x}^{2}+k_{y}^{2}}\right)^{\alpha-1}}{\sum_{k_{x} \in k_{\mathrm{res}}} \sum_{k_{y} \in k_{\mathrm{res}}} \psi\left(\sqrt{k_{x}^{2}+k_{y}^{2}}\right)^{\alpha-1}}
\end{aligned}
$$

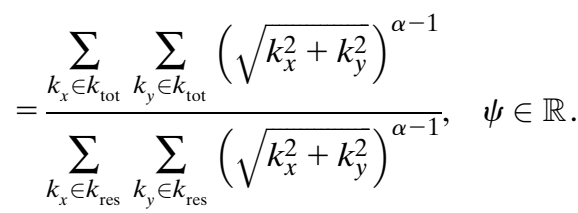

With this an estimate for the unresolved part of the variance due to lower wavenumbers depending on the assumed exponent $\alpha$ can be obtained:

$$
\left[\sigma_{\text {unres }}^{2}(\alpha)\right]^{\mathrm{est}}:=\frac{\sum_{k_{x} \in k_{\mathrm{tot}}} \sum_{k_{y} \in k_{\mathrm{tot}}}\left(\sqrt{k_{x}^{2}+k_{y}^{2}}\right)^{\alpha-1}}{\sum_{k_{x} \in k_{\mathrm{res}}} \sum_{k_{y} \in k_{\mathrm{res}}}\left(\sqrt{k_{x}^{2}+k_{y}^{2}}\right)^{\alpha-1} \sigma_{\mathrm{res}}^{2}-\sigma_{\mathrm{res}}^{2} .}
$$

The exponent $\alpha$, which minimizes the following difference of the correct unresolved variance, which can be calculated by integrating over the respective wavenumbers and the one estimated by extrapolation is the resulting best estimate for the exponent of the approximated power law:

$$
\begin{aligned}
{\left[\sigma_{\text {unres }}^{2}(\alpha)\right]^{\text {est }}-\sigma_{\text {unres }}^{2}=} & {\left[\sigma_{\text {unres }}^{2}(\alpha)\right]^{\text {est }} } \\
& -\sum_{k_{x} \in k_{\text {unres }}} \sum_{k_{y} \in k_{\text {urres }}} \mathcal{P}\left(k_{x}, k_{y}\right) .
\end{aligned}
$$

In this evaluation $3 / 4$ of the intermediate wavenumber space is defined as resolved and the remaining $1 / 4$ is defined as unresolved. The result of this evaluation can be seen in Fig. 9 and Table 3.

The results for the large-scale models (GCM and NWP) from section $3 \mathrm{c}$ are broadly confirmed. There 

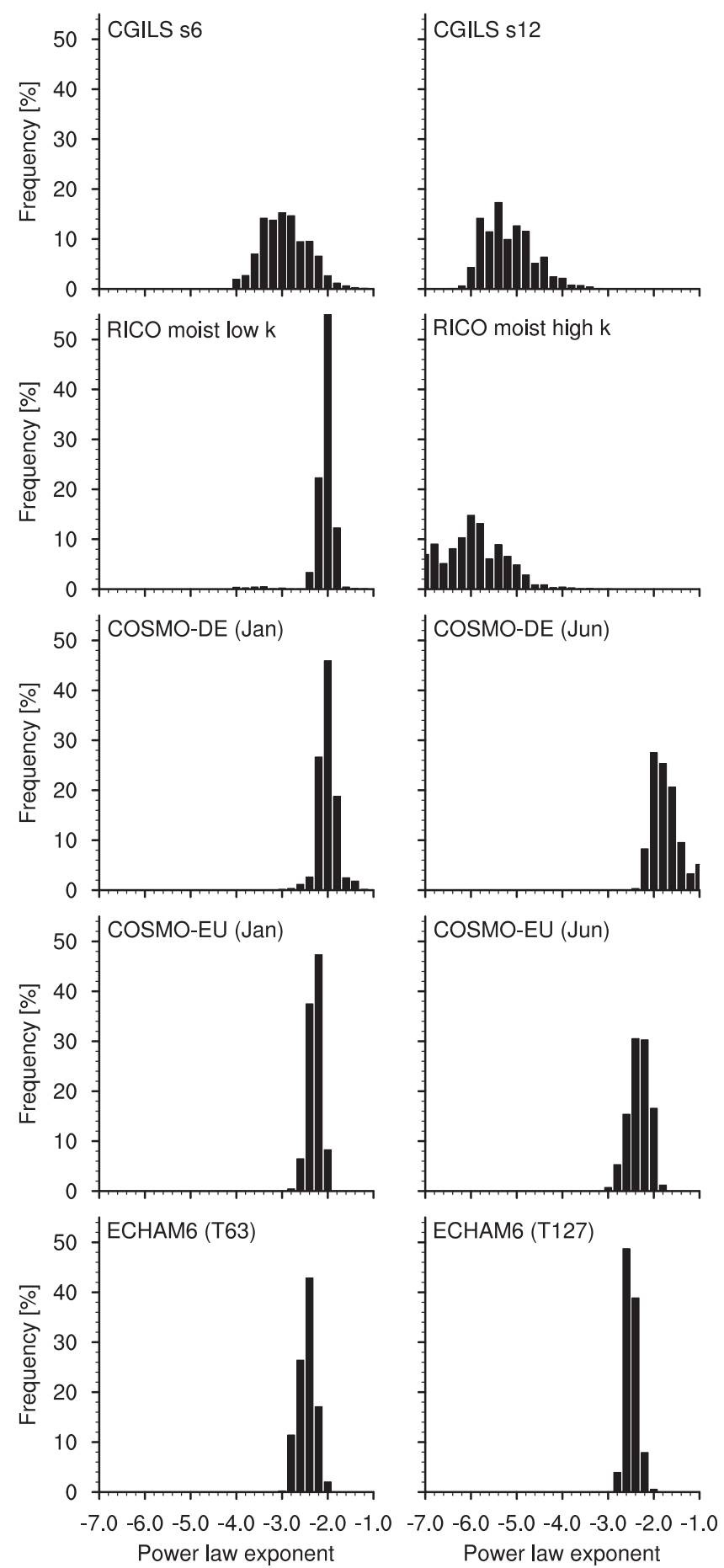

FIG. 9. As in Fig. 8, except estimating the power-law exponents using the extrapolation approach.

are small variations but these are likely attributable to different methods. The mean values and the shape of the histograms still show a consistent scaling for all largescale models. Here, the respective mean exponent for the large-scale models is $\alpha=-2.3 \pm 0.33$, which is well within the range of uncertainty of the exponent derived from the previous approach. The LES cases instead show again a lot more variability and higher mean values 
TABLE 3. As in Table 2, but using the approach based on extrapolation.

\begin{tabular}{lcc}
\hline \hline \multicolumn{1}{c}{ Data } & $\begin{array}{c}\text { Mean } \\
\text { exponent }(\alpha)\end{array}$ & $\begin{array}{c}\text { Standard } \\
\text { deviation }\end{array}$ \\
\hline LES (CGILS s12) & -5.18 & \pm 0.54 \\
LES (CGILS s6) & -2.92 & \pm 0.50 \\
LES (RICO moist low $k$ ) & -2.04 & \pm 0.26 \\
LES (RICO moist high $k$ ) & -5.93 & \pm 0.67 \\
NWP (COSMO-DE January) & -2.00 & \pm 0.21 \\
NWP (COSMO-DE June) & -1.73 & \pm 0.29 \\
NWP (COSMO-EU January) & -2.29 & \pm 0.16 \\
NWP (COSMO-EU June) & -2.33 & \pm 0.24 \\
GCM (ECHAM6 T63) & -2.47 & \pm 0.17 \\
GCM (ECHAM6 T127) & -2.51 & \pm 0.13 \\
\hline
\end{tabular}

than in section 3c. Again, this high uncertainty might be difficult to be explained by physical reasons and caused by other issues. As increasingly large-domain and finegrid-resolution simulations become available these issues should become easier to resolve.

\section{Parameterizations of variance}

\section{a. Statistical cloud cover scheme}

One example of a statistical cloud cover scheme is the one from Tompkins (2002), which is part of the ECHAM6 GCM. In this scheme, the total water mixing ratio is assumed to be described by a beta distribution. The upper and lower bounds of the distribution are described by two parameters $(a, b)$. Two additional parameters $(p, q)$ define the skewness. Instead of using prognostic equations for the moments of the distribution directly, Tompkins (2002) used the distribution width $(b-a)$ and the skewness parameter $q$ as prognostic equations and for simplicity, assumed $p$ to be constant $(p=2)$. The two prognostic equations are described by source and sink terms due to turbulence $(t)$, convection $(c)$, and microphysics $(m)$ :

$$
\begin{aligned}
\frac{\partial(b-a)}{\partial t} & =\left[\frac{\partial(b-a)}{\partial t}\right]_{c}+\left[\frac{\partial(b-a)}{\partial t}\right]_{t} \\
\frac{\partial q}{\partial t} & =\left(\frac{\partial q}{\partial t}\right)_{c}+\left(\frac{\partial q}{\partial t}\right)_{t}+\left(\frac{\partial q}{\partial t}\right)_{m} .
\end{aligned}
$$

To calculate the three free parameters- $a, b$, and $q-$ three equations are needed. For partial cloudiness the mean values of total water and cloud water mixing ratio and the prognostic equation for $q$ are used. In the case of cloud fraction zero (clear sky) or one (overcast sky), the prognostic distribution width $(b-a)$ is used instead of the mean cloud water mixing ratio.
Ideally, either through the coupling to other parameterizations, or by direct specification, the cloud cover scheme would get information about the resolution and be adapted to different grid sizes. As seen in the previous sections this should lead to a scaling behavior of the variance of total water mixing ratio. Variance is not a prognostic variable in the original formulation of Tompkins (2002), but can be calculated by using the distribution width $(b-a)$ and the shape parameters $(p, q)$ as follows:

$$
\overline{r_{t}^{\prime 2}}=\frac{(b-a)^{2}}{(p+q)^{2}} \frac{p q}{p+q+1} .
$$

For the evaluation of the statistical cloud cover scheme in the following section, Eq. (20) is used to calculate the parameterized subgrid-scale variance of totalwater mixing ratio. With this not only the prognostic equation for the distribution width [Eq. (18)] is evaluated, but error and problems caused by both prognostic equations [Eqs. (18) and (19)] are included in the evaluation of the variance [Eq. (20)].

\section{b. Evaluation of the parameterized variance of total- water mixing ratio}

In this section the parameterized subgrid-scale variance of total water mixing ratio, which is predicted by the statistical cloud scheme of Tompkins (2002), is evaluated with regard to scale dependency. For this evaluation the output of the ECHAM6 setup as described in section $2 \mathrm{a}$ is used and the parameterized subgrid-scale variance is calculated by Eq. (20) using the prognostic distribution width, the prognostic shape parameter $q$, and the fixed shape parameter $p=2$. For comparison, another subgrid-scale variance is calculated by extrapolating the resolved T63 variance with a power-law exponent of $\alpha=$ 2.1 , which is the mean value of the exponents estimated for the large-scale models (GCM and NWP) with both methods. The extrapolation is done with the method described in section $3 \mathrm{~d}$ up to a maximum wavenumber of $k_{\max }=10^{-4} \mathrm{~m}^{-1}$, which corresponds to a resolution of $10 \mathrm{~km}$. Because of the already mentioned numerical constraints, only the intermediate wavenumber range can be used for the extrapolation. This leads to an uncertainty in the comparison of the different variances, as actually more variance is resolved by the model than used for the extrapolation. But the main results are not affected by this uncertainty.

In Table 4 the mean values of the resolved, the extrapolated subgrid-scale, and the parameterized subgrid-scale variance for the two different ECHAM6 resolutions T63 and T127 are shown. The total variancethe sum of resolved variance and the mean subgrid-scale 
TABLE 4. Mean values of resolved, extrapolated unresolved, and parameterized unresolved variance in simulations of the ECHAM6 GCM at T63 $(\approx 190 \mathrm{~km})$ and T127 $(\approx 100 \mathrm{~km})$ horizontal resolutions. The term "resolved" refers to the amount of variance resolved by the mentioned resolution. The extrapolated part is the amount of unresolved variance extrapolated under the assumption of a power law of $\alpha=-2.1$. The parameterization of the subgridscale variability (parameterized) is the one by Tompkins (2002).

\begin{tabular}{lccc}
\hline & $\mathrm{T} 63$ & $\mathrm{~T} 127$ & $\mathrm{~T} 127-\mathrm{T} 63$ \\
\hline Resolved $\left(\mathrm{kg} \mathrm{kg}^{-1}\right)^{2}$ & $2.92 \times 10^{-7}$ & $4.89 \times 10^{-7}$ & $+1.97 \times 10^{-7}$ \\
$\begin{array}{l}\text { Extrapolated } \\
\left(\mathrm{kg} \mathrm{kg}^{-1}\right)^{2}\end{array}$ & $1.58 \times 10^{-7}$ & $9.76 \times 10^{-8}$ & $-6.01 \times 10^{-8}$ \\
$\begin{array}{c}\text { Parameterized } \\
\left(\mathrm{kg} \mathrm{kg}^{-1}\right)^{2}\end{array}$ & $7.74 \times 10^{-8}$ & $7.58 \times 10^{-8}$ & $-1.59 \times 10^{-9}$ \\
\hline
\end{tabular}

variance-should not change with changing resolutions. This means, that with an increasing resolved varianceT127 compared to T63-the subgrid-scale variance should decrease. As shown in Table 4, neither the sum of resolved variance and parameterized subgrid-scale variance nor the sum of resolved variance and extrapolated subgrid-scale variance is really constant. But the differences are remarkable higher for the parameterized subgrid-scale variance. Table 4 shows also for the absolute values that the amount of parameterized subgrid-scale variance is smaller than the one calculated by extrapolating. This is consistent with earlier results of, for example, Quaas (2012) and Weber et al. (2011), which stated that the parameterized subgridscale variance of total water mixing ratio in the scheme of Tompkins (2002) is underestimated.

More remarkable is the missing scale dependency of the parameterized subgrid-scale variance. The amount of parameterized subgrid-scale variance should decrease with increasing resolution, but while this effect can be seen for the extrapolated variance, the parameterized variance does not decrease significantly. More details on the changes are provided by the histograms (Fig. 10) of the differences for the resolved, the extrapolated subgrid-scale, and the parameterized subgrid-scale variances. For the resolved variance, the values are all positive, as with the higher T127 resolution more variance is resolved than with the coarser T63 (Fig. 10a). Respectively, the difference values for the extrapolated subgrid-scale variances are negative (Fig. 10b), as the subgrid-scale variance should decrease with increasing resolution. But the values for the parameterized subgridscale variances are smaller in magnitude and have both signs: negative and positive (Fig. 10c). Both the smaller magnitude and the mix of signs show clearly that the variance parameterized by the Tompkins scheme is missing a significant scale dependency. This explains why the mean difference between both resolutions of the
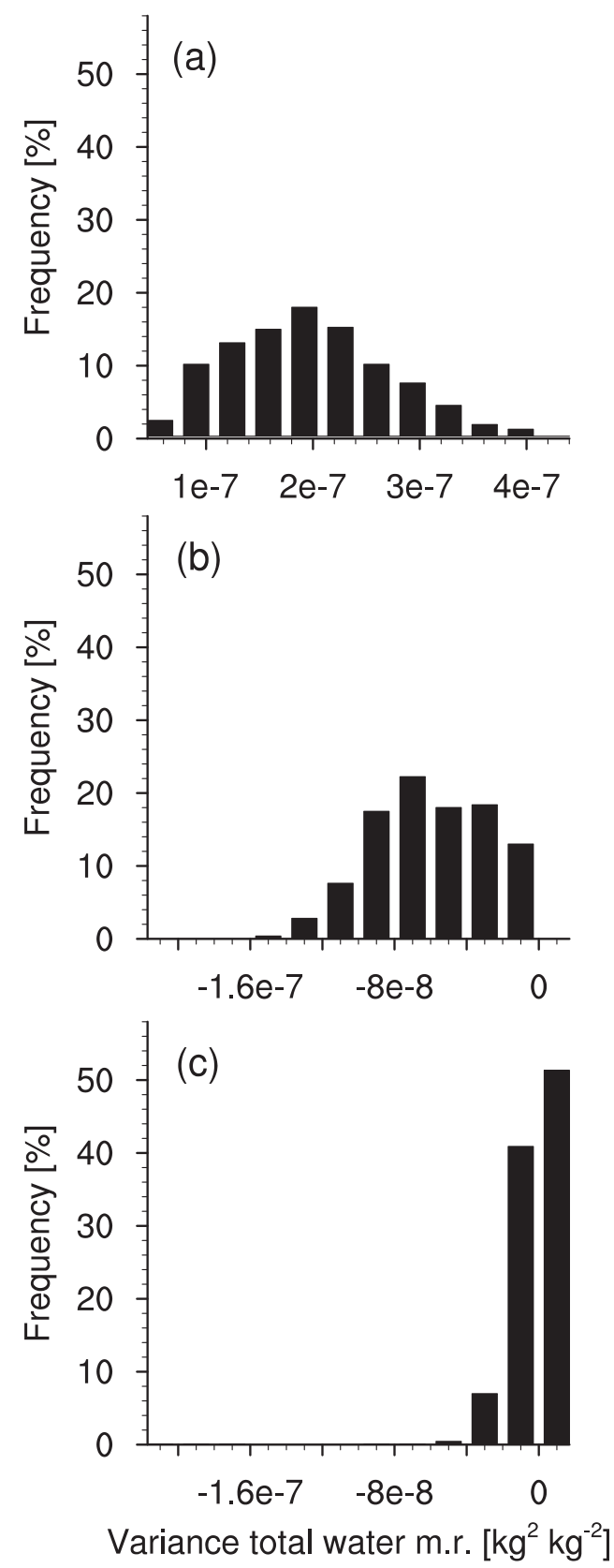

FIG. 10. Histograms of the difference (T127 - T63) for (a) the resolved, (b) the extrapolated subgrid-scale, and (c) the parameterized subgrid-scale variance, one value for each time step and each height.

parameterized variance (see Table 4) is two orders of magnitude smaller than expected.

The vertical structure of the three different types of variances is shown in Fig. 11. This time the mean exponents for the large-scale models from both approaches and their standard deviations $(\alpha=-2.3 \pm 0.33$ and $\alpha=-1.94 \pm 0.24)$ are used for the extrapolation. This 

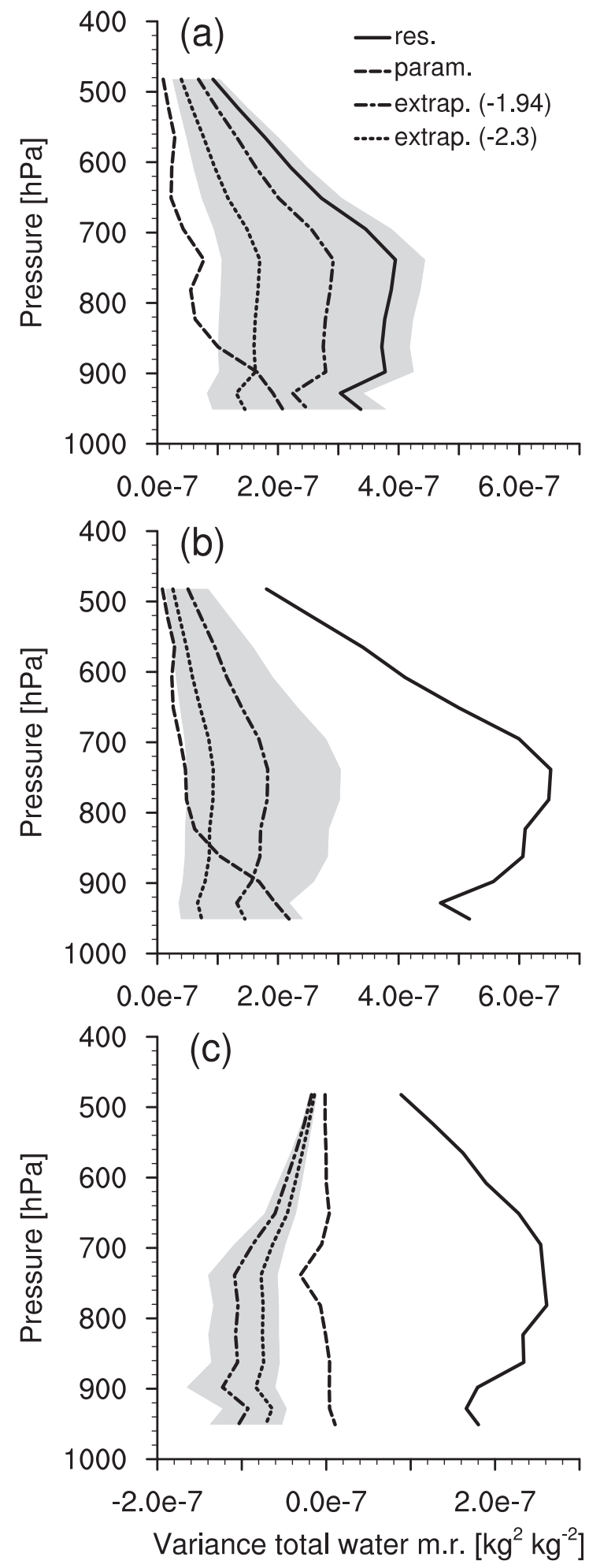

FIG. 11. Vertical profiles of resolved (solid), extrapolated subgrid-scale (dotted, dashed-dotted), and parameterized subgridscale (dashed) variance of (a) the T63, (b) the T127 resolution, and (c) the difference (T127 - T63). The gray shaded area represents the standard deviations for both estimated exponents. produces some estimate for the spread in the variance, which is caused by the uncertainty in the exponents. The vertical profiles confirm the underestimation of the amount (Figs. 11a,b) and scale dependency (Fig. 11c) of the parameterized subgrid-scale variability by the Tompkins scheme. Additionally, the vertical profile of the parameterized subgrid-scale variance shows a strong decrease in the lower troposphere, which is in contrast to the constant or slightly increasing profile of the resolved variance. This gives some evidence on an unphysical behavior of the parameterized subgrid-scale variance, which depends on the parameterized distribution width [Eq. (18)] and shape parameter $q$ [Eq. (19)].

\section{Conclusions}

Three different kinds of models (GCM, NWP, and LES) covering a broad range of scales were analyzed to estimate the scale dependency of variance of total water mixing ratio. The combined power density spectrum showed a consistent and continuous scaling from global scales down to the small LES scales. The analysis provides no evidence for a spectral gap, nor is there a clear signal of a strong break in the scaling of the spectra. However, because of an increased variability of the estimated power-law exponent at small scales, a weak scale break toward $-5 / 3$ or $-7 / 3$ cannot be ruled out by our analysis. The existence of clear scaling at all investigated scales implies the necessity to include a scale dependency in future cloud process parameterizations.

For the large-scale models (GCM and NWP) a common scaling exponent of $\alpha \approx-2$ could be estimated. As the NWP is covering the scales mainly contributing to the unresolved variance in a current GCM, this estimate provides a sufficiently good approximation for the evaluation of subgrid-scale parameterizations. The exponent -2 also lies in the range of earlier estimates of a scaling exponent of water vapor. Because the influence of cloud water and ice on the scaling is rather small for the large scales, the estimates of previous studies on water vapor and the result of this study show a relatively good agreement.

For the smaller scales (less than $\sim 1 \mathrm{~km}$ ) more variability and a decreasing exponent at the higher wavenumbers were discovered. The change of scaling during the scales of a typical LES model could hint at a scale break. But as a scaling exponent of $\alpha \approx-2$ was also found in DNS data, this change in the scaling is likely due to excessive dissipation in individual models rather than evidence of a strong break in scaling. Looking from a global modeling perspective the variance produced at scales less than $1 \mathrm{~km}$ is still rather negligible. Nevertheless, the discovered variability merits more 
attention, as does the possibility of a slight change in scaling at small scales.

The evaluation of the parameterized variance in the statistical scheme of Tompkins (2002) showed an underestimation of the amount of subgrid-scale variance in the current formulation. But more remarkable is the nonexistent scale dependency of the parameterized subgrid-scale variance of total water mixing ratio. As a follow up, the explored scale dependency should be included in the parameterized variance to receive an improved representation of subgrid-scale variability at different resolutions. The common scaling behavior of total water variance at a broad range of scales explored in this study underlines the importance of the development of scale-aware parameterizations also for current GCMs.

Acknowledgments. This project is funded by the extramural research program of the German Weather Service (Deutscher Wetterdienst) and the Max Planck Society for Advancement of Science. The authors thank Axel Seifert and Thijs Heus for providing the LES data and helpful discussions. Thanks also to Alberto de Lozar for providing the DNS data. Jürgen Bader and four anonymous reviewers are thanked for their comments on this manuscript. Discussions during the COST Activity ES0905 on "Basic concepts for convection parameterization in weather forecast and climate models" gave additional input to the presented study.

\section{REFERENCES}

André, J. C., G. D. Moor, P. Lacarrre, and R. D. Vachat, 1976: Turbulence approximation for inhomogeneous flows: Part I. The clipping approximation. J. Atmos. Sci., 33, 476-481.

Arakawa, A., J.-H. Jung, and C.-M. Wu, 2011: Toward unification of the multiscale modeling of the atmosphere. Atmos. Chem. Phys., 11, 3731-3742, doi:10.5194/acp-11-3731-2011.

Baldauf, M., A. Seifert, J. Foerstner, D. Majewski, M. Raschendorfer, and T. Reinhardt, 2011: Operational convective-scale numerical weather prediction with the COSMO model: Description and sensitivities. Mon. Wea. Rev., 139, 3887-3905.

Blossey, P. N., and Coauthors, 2013: Marine low cloud sensitivity to an idealized climate change: The CGILS LES intercomparison. J. Adv. Model. Earth Syst., 5, 234-258, doi:10.1002/ jame.20025.

Bony, S., and J.-L. Dufresne, 2005: Marine boundary layer clouds at the heart of tropical cloud feedback uncertainties in climate models. Geophys. Res. Lett., 32, L20806, doi:10.1029/ 2005 GL023851.

Cho, J. Y. N., R. E. Newell, and G. W. Sachse, 2000: Anomalous scaling of mesoscale tropospheric humidity fluctuations. Geophys. Res. Lett., 27, 377-380.

Cusack, S., J. M. Edwards, and R. Kershaw, 1999: Estimating the subgrid variance of saturation, and its parametrization for use in a GCM cloud scheme. Quart. J. Roy. Meteor. Soc., 125, 3057-3076, doi:10.1002/qj.49712556013.

Fischer, L., C. Kiemle, and G. C. Craig, 2012: Height-resolved variability of midlatitude tropospheric water vapor measured by an airborne lidar. Geophys. Res. Lett., 39, L06803, doi:10.1029/ 2011GL050621.

Hourdin, F., and Coauthors, 2006: The LMDZ4 general circulation model: climate performance and sensitivity to parametrized physics with emphasis on tropical convection. Climate Dyn., 27, 787-813, doi:10.1007/s00382-006-0158-0.

Kahn, B. H., and J. Teixeira, 2009: A global climatology of temperature and water vapor variance scaling from the atmospheric infrared sounder. J. Climate, 22, 5558-5576.

_ , and Coauthors, 2011: Temperature and water vapor variance scaling in global models: Comparisons to satellite and aircraft data. J. Atmos. Sci., 68, 2156-2168.

Klein, S. A., R. Pincus, C. Hannay, and K.-M. Xu, 2005: How might a statistical cloud scheme be coupled to a mass-flux convection scheme? J. Geophys. Res., 110, D15S06, doi:10.1029/ 2004JD005017.

Larson, V. E., and J.-C. Golaz, 2005: Using probability density functions to derive consistent closure relationships among higher-order moments. Mon. Wea. Rev., 133, 1023-1042.

Mellado, J. P., 2010: The evaporatively driven cloud-top mixing layer. J. Fluid Mech., 660, 5-36, doi:10.1017/S0022112010002831.

Nastrom, G. D., W. H. Jasperson, and K. S. Gage, 1986: Horizontal spectra of atmospheric tracers measured during the global atmospheric sampling program. J. Geophys. Res., 91 (D12), 13201-13209.

NCL, 2012: The NCAR Command Language (version 6.0.0). UCAR/NCAR/CISL/VETS. [Available online at http://dx. doi.org/10.5065/D6WD3XH5.]

Pressel, K. G., and W. D. Collins, 2012: First-order structure function analysis of statistical scale invariance in the AIRSobserved water vapor field. J. Climate, 25, 5538-5555.

Quaas, J., 2012: Evaluating the "critical relative humidity" as a measure of subgrid-scale variability of humidity in general circulation model cloud cover parameterizations using satellite data. J. Geophys. Res., 117, D09208, doi:10.1029/ 2012JD017495.

Randall, D. A., and Coauthors, 2007: Climate models and their evaluation. Climate Change 2007: The Physical Science Basis, S. Solomon et al., Eds., Cambridge University Press, 589-662.

Rauber, R. M., and Coauthors, 2007: Rain in shallow cumulus over the ocean: The RICO campaign. Bull. Amer. Meteor. Soc., 88, 1912-1928.

Satoh, M., T. Matsuno, H. Tomita, H. Miura, T. Nasuno, and S. Iga, 2008: Nonhydrostatic icosahedral atmospheric model (NICAM) for global cloud resolving simulations. J. Comput. Phys., 227, 3486-3514, doi:10.1016/j.jcp.2007.02.006.

Seifert, A., and T. Heus, 2013: Large-eddy simulation of organized precipitating trade wind cumulus clouds. Atmos. Chem. Phys. Discuss., 13, 1855-1889, doi:10.5194/acpd-13-1855-2013.

Stevens, B., and A. Seifert, 2008: On the sensitivity of simulations of shallow cumulus convection to their microphysical representation. J. Meteor. Soc. Japan, 86A, 143-162.

— C.-H. Moeng, and P. P. Sullivan, 1999: Large-eddy simulations of radiatively driven convection: Sensitivities to the representation of small scales. J. Atmos. Sci., 56, 39633984. 
- and Coauthors, 2005: Evaluation of large-eddy simulations via observations of nocturnal marine stratocumulus. Mon. Wea. Rev., 133, 1443-1462.

- and Coauthors, 2013: The atmospheric component of the MPI-M Earth System Model: ECHAM6. J. Adv. Model. Earth Syst., 5, 146-172, doi:10.1002/jame.20015.

Tompkins, A. M., 2002: A prognostic parameterization for the subgrid-scale variability of water vapor and clouds in largescale models and its use to diagnose cloud cover. J. Atmos. Sci., 59, 1917-1942.
_ 2008: Cloud parametrization. Proc. ECMWF Seminar on Parametrization of Subgrid Physical Processes, Shinfield Park, United Kingdom, ECMWF, 27-62.

Wan, H., and Coauthors, 2013: The ICON-1.2 hydrostatic atmospheric dynamical core on triangular grids-Part 1: Formulation and performance of the baseline version. Geosci. Model. Dev. Discuss., 6, 59-119, doi:10.5194/gmdd-6-59-2013.

Weber, T., J. Quaas, and P. Räisänen, 2011: Evaluation of the statistical cloud scheme in the ECHAM5 model using satellite data. Quart. J. Roy. Meteor. Soc., 137, 2079-2091, doi:10.1002/qj.887. 